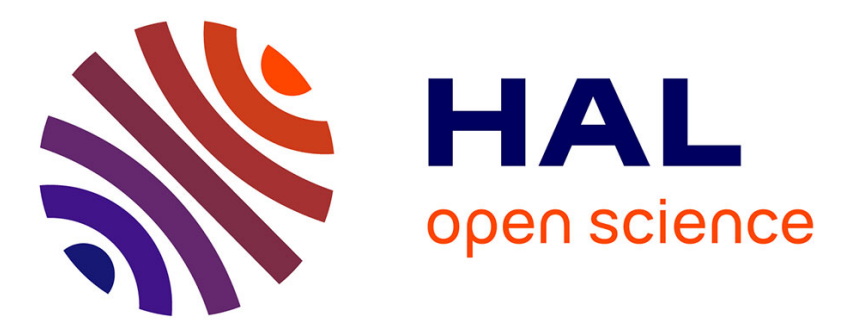

\title{
Interior Structure Models of Solid Exoplanets Using Material Laws in the Infinite Pressure Limit
}

\author{
F.W. Wagner, F. Sohl, H. Hussmann, M. Grott, H. Rauer
}

\section{To cite this version:}

F.W. Wagner, F. Sohl, H. Hussmann, M. Grott, H. Rauer. Interior Structure Models of Solid Exoplanets Using Material Laws in the Infinite Pressure Limit. Icarus, 2011, 214 (2), pp.366. 10.1016/j.icarus.2011.05.027 . hal-00786873

\section{HAL Id: hal-00786873 https://hal.science/hal-00786873}

Submitted on 11 Feb 2013

HAL is a multi-disciplinary open access archive for the deposit and dissemination of scientific research documents, whether they are published or not. The documents may come from teaching and research institutions in France or abroad, or from public or private research centers.
L'archive ouverte pluridisciplinaire HAL, est destinée au dépôt et à la diffusion de documents scientifiques de niveau recherche, publiés ou non, émanant des établissements d'enseignement et de recherche français ou étrangers, des laboratoires publics ou privés. 


\section{Accepted Manuscript}

Interior Structure Models of Solid Exoplanets Using Material Laws in the Infinite Pressure Limit

F.W. Wagner, F. Sohl, H. Hussmann, M. Grott, H. Rauer

PII: S0019-1035(11)00200-4

DOI: 10.1016/j.icarus.2011.05.027

Reference: YICAR 9834

To appear in:

Icarus

Received Date: $\quad 15$ December 2010

Revised Date: $\quad 5$ May 2011

Accepted Date: $\quad 18$ May 2011

Please cite this article as: Wagner, F.W., Sohl, F., Hussmann, H., Grott, M., Rauer, H., Interior Structure Models of Solid Exoplanets Using Material Laws in the Infinite Pressure Limit, Icarus (2011), doi: 10.1016/j.icarus. 2011.05.027

This is a PDF file of an unedited manuscript that has been accepted for publication. As a service to our customers we are providing this early version of the manuscript. The manuscript will undergo copyediting, typesetting, and review of the resulting proof before it is published in its final form. Please note that during the production process errors may be discovered which could affect the content, and all legal disclaimers that apply to the journal pertain. 


\title{
Interior Structure Models of Solid Exoplanets Using Material Laws in the Infinite Pressure Limit
}

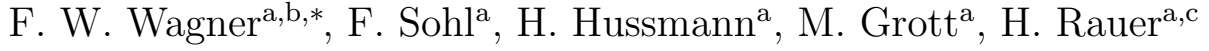 \\ ${ }^{a}$ Institute of Planetary Research, German Aerospace Center (DLR), Berlin, Germany \\ ${ }^{b}$ Institute for Planetology, Westphalian Wilhelms-University, Münster, Germany \\ ${ }^{c}$ Center of Astronomy and Astrophysics, Technical University of Berlin, Berlin, Germany
}

\section{Abstract}

The detection of low-mass extrasolar planets has initiated growing interest in massive rocky bodies (super-Earths) for which no solar system analogue does exist. Here, we present a new model approach to investigate their interior structure and thermal state. We improve and extend previous interior models mainly in two areas: The first improvement is due to the consequent application of equations of state (EoS) that are compliant with the thermodynamics of the high-pressure limit and facilitate reinvestigating mass-radius relations for terrestrial-type exoplanets. To quantify the uncertainty due to extrapolation, we compare a generalized Rydberg and a Keane EoS, which are both consistent with the physics of the thermodynamic limit. Furthermore, we consider a reciprocal $K^{\prime}$ EoS that fits the seismologically obtained Preliminary Reference Earth Model (PREM), thereby accounting for the mineralogical composition of the Earth. As a result, the predicted planetary radii of terrestrial-type ex-

*Corresponding author; Tel: +49 3067055529 // Fax: +49 3067055303

Email address: frank.wagner@dlr.de (F. W. Wagner) 
oplanets of up to ten Earth masses would differ by less than $2 \%$ between all three EoS, well within current observational limits. The second extension arises from the adoption of a mixing length formulation instead of the commonly used, more simplified parameterized approach to model convective heat transport in planetary mantles. In comparison to parameterized convection models, our results indicate generally hotter interiors with increasing planetary mass and a cumulative tendency to extended regimes of sluggish convection in the lowermost mantle. The latter is attributed to less efficient convective heat transport with increasing mantle pressures. An improved knowledge of the present thermal state is prerequisite to gain a better understanding of the pathways of internal evolution of terrestrial-type exoplanets. Keywords: super-Earths, interior structure, thermal state, mass-radius relationships, high-pressure physics

\section{Introduction}

2 Owing to the growing number of detected exoplanets, the field of compar3 ative exoplanetology is rapidly expanding since the first discovery of a planet 4 orbiting a star other than the Sun. More than 30 exoplanets with masses 5 below 15 Earth masses $\left(\mathrm{M}_{\oplus}\right)$ have been discovered and without a doubt more 6 will be detected by either space missions or ground-based surveys. One of 7 the most prominent low-mass exoplanets is CoRoT-7b (Léger et al., 2009), \& which is supposed to be the first discovered rocky exoplanet or super-Earth (Queloz et al., 2009). Another example is GJ 1214b (Charbonneau et al., 2009), a planet with a total mass of $(6.55 \pm 0.98) \mathrm{M}_{\oplus}$ and a planetary radius of $(2.68 \pm 0.13) R_{\oplus}$, implying a relatively low mean density without solar 

35200 GPa to 10 TPa (Seager et al., 2007; Grasset et al., 2009), we 36 consider different EoS (generalized Rydberg, Keane, and recipro-

system analogue (Rogers and Seager, 2010). Also worth mentioning is the planetary system of Gliese 581 containing at least four planets: Including Gliese 581d (Mayor et al., 2009), a low-mass exoplanet likely situated within the habitable zone, a region where liquid water may exist on the planetary surface, and Gliese 581e (Mayor et al., 2009), an exoplanet with the smallest currently known minimum mass of about $2 \mathrm{M}_{\oplus}$ orbiting a main sequence star. These are only a few examples of the remarkable variety of low-mass exoplanets detected so far.

Previous work by Valencia et al. (2006, 2007a,b), Sotin et al. (2007), Fortney et al. (2007), Seager et al. (2007), and Grasset et al. (2009) have investigated mass-radius relations for silicate- and water-rich exoplanets using different bulk compositions, equations of state (EoS), and other modeling details. Uncertainties and discrepancies of such models mainly result from the necessity to extrapolate an EoS to high pressures and, to a minor extent, from the lack of knowledge about internal heat sources and rheological properties that would determine the present thermal state of exoplanet interiors. The majority of the EoS used (e.g., Birch-Murnaghan, Vinet) represent semiempirical fits obtained from laboratory experiments and are not essentially consistent with the thermodynamics of the high-pressure limit (Stacey and Davis, 2004). Nevertheless, interior structure models for exoplanets have been calculated based on the Vinet EoS (e.g., Valencia et al., 2007a) or the Birch-Murnaghan EoS (e.g., Sotin et al., 2007). As a consequence of the lack of reliable experimental data mainly in the pressure range of 
cal $K^{\prime}$ ) that are consistent with the physics of the thermodynamic

limit. Hence, the present study is aimed at a comparison between thermodynamically consistent EoS to investigate how these would affect interior structure models of massive terrestrial-type exoplanets. Another purpose of this study is to apply the concept of mixing length to self-consistently model the present thermal state of planetary interiors. Therefore, compared to previous models, the main differences are related to the EoS used in this study and how radial temperature profiles of model planets are obtained.

In the following section, we describe how the interior structure models and corresponding radial temperature profiles are constructed and relevant EoS parameters are obtained. In section 3, we present our results for the implemented EoS and quantify their effects on the structural models of exoplanet interiors. Furthermore, radial temperature profiles calculated by using the adopted mixing length approach are presented. Additionally, mass-radius relations for silicate- and water-rich exoplanets are used to validate our model approach. In section 4, we discuss our results obtained from the EoS comparison and the mixing length formulation by addressing main differences to previous models and implications for the robustness of mass-radius relations. Finally, conclusions are drawn and possible consequences for observational tresholds of present and future missions will be summarized.

\section{Method}

We model the interior structure and calculate mass-radius relations for several classes of low-mass exoplanets. 


\subsection{Bulk Composition and Phase Transitions}

In the present study, we consider three principal classes of low-mass exoplanets according to their bulk compositions: (a) Terrestrial planets such as the Earth and Venus, for which an Earth-like bulk composition with an iron core mass fraction of 32.5 wt.- $\%$ and a corresponding silicate mantle mass fraction of 67.5 wt.- $\%$ are assumed. This class of exoplanets is of particular interest because of the known potential to be life-sustaining. (b) Iron-rich Mercury-type planets containing 70 wt.- $\%$ iron, which is concentrated in the core, and a silicate mantle of 30 wt.-\%. (c) Ocean planets are assumed to contain at least $10 \% \mathrm{H}_{2} \mathrm{O}$ by mass (Léger et al., 2004) and have bulk compositions similar to that of a cometary core $(50 \mathrm{wt} .-\%$ volatiles and $50 \mathrm{wt}$. $\%$ rock and iron). In the following, we consider an ocean planet similar to Jupiter's icy moon Ganymede, which is composed of 45 wt.- $\%$ water-ice, 48.5 wt.- $\%$ silicate, and 6.5 wt.-\% iron (Kuskov and Kronrod, 2001; Sohl et al., 2002).

Each rocky planet is subdivided into four chemically homogeneous shells. The mantle consists of an upper olivine part composed of forsterite, underlain by a perovskite shell and a lower post-perovskite part, whereas the central core is assumed to be composed of pure iron. Therefore, two pressureinduced phase transitions have been implemented into the model: (a) the olivine to perovskite and (b) the perovskite to post-perovskite transition are experimentally determined with Clapeyron slopes of $-0.0013 \mathrm{MPa} \mathrm{K}^{-1}$ (Fei et al., 2004) and +13.3 MPa $\mathrm{K}^{-1}$ (Tateno et al., 2009), respectively. We have chosen a relatively large value for the Clapeyron slope of the perovskite to post-perovskite transition in accordance with the seismic discontinuity observed in the Earth's D" region (Hernlund and Labrosse, 2007). First 
principles calculations suggest that post-perovskite could dissociate into CsCl-type $\mathrm{MgO}$ and cotunnite-type $\mathrm{SiO}_{2}$ at pressures and temperatures prevalent in the deep interiors of rocky exoplanets (Umemoto et al., 2006). However, since only a small volume of the hot, lowermost mantle near the core-mantle boundary of Earthlike exoplanets would be affected by the small density increase at pressures above $1 \mathrm{TPa}$, it can be safely assumed that neglecting the putative dissociation of post-perovskite will not have a severe impact on the radial mass distribution.

In the case of ocean planets, an additional high-pressure water-ice layer is placed on top of the silicate mantle. We neglect low-pressure phase transitions within the ice because of their minor effect on mass-radius relations (Seager et al., 2007). In addition to the core mass fraction, we finally have to specify the water mass fraction for ocean planets.

\subsection{Numerical Model}

We consider a spherically symmetric and fully differentiated planet in thermal steady state and perfect mechanical equilibrium. Under these assumptions, its depth-dependent interior structure is described by the following set of coupled differential equations for mass $m(r)$, acceleration of gravity $g(r)$, and pressure $P(r)$ :

$$
\begin{aligned}
\frac{\mathrm{d} m}{\mathrm{~d} r} & =4 \pi r^{2} \rho, \\
\frac{\mathrm{d} g}{\mathrm{~d} r} & =4 \pi \mathrm{G} \rho-2 \frac{g}{r}, \\
\frac{\mathrm{d} P}{\mathrm{~d} r} & =-\rho g,
\end{aligned}
$$


where $r$ is the radial distance from the center of the planet, $G$ is the gravitational constant, and $\rho$ is the local density described in detail in the next paragraph.

Within the core, we assume an adiabatic temperature distribution given by

$$
\frac{\mathrm{d} T}{\mathrm{~d} r}=-\frac{\gamma}{\Phi} g T,
$$

where $\gamma$ is the thermodynamic Grüneisen parameter and $\Phi=K_{S} / \rho$ the seismic parameter. The latter involves the adiabatic bulk modulus $K_{S}$ and density $\rho$, which are both obtained from the local evaluation of an equation of state. The equation $K_{S} / K_{T}=1+\gamma \alpha T$ relates the adiabatic bulk modulus $K_{S}$ to the isothermal bulk modulus $K_{T}$, where $\alpha$ is the thermal expansivity of a given material. No additional heat sources are assumed in the core. Nevertheless, we evaluate a minimum heat flux conducted along the core adiabat across the core-mantle boundary $(\mathbf{c m b})$ of $q_{c m b}=-k d T / d r$ at $r=r_{c m b}$, where $k$ is the thermal conductivity of the core taken constant at $35 \mathrm{~W} \mathrm{~K}^{-1} \mathbf{m}^{-1}$ (Stacey and Davis, 2008).

Within the silicate mantle, the following equations are considered for heat flux $q(r)$ and temperature $T(r)$ as functions of the radial distance $r$ :

$$
\begin{aligned}
\frac{\mathrm{d} q}{\mathrm{~d} r} & =\epsilon \rho-2 \frac{q}{r} \\
\frac{\mathrm{d} T}{\mathrm{~d} r} & =-\frac{q}{N u_{r} k_{c}}
\end{aligned}
$$



according to

where $N u_{r}$ is the dimensionsless local Nusselt number and $k_{c}$ is the thermal conductivity of the mantle as given by the model of Hofmeister (1999). For the sake of simplicity, we limit ourselves to radiogenic heating; accretional heating and secular cooling or tidal heating are therefore not explicitly taken into account. The specific heat production rate $\epsilon$ is taken to be constant and matches the present-day Earth-like value of $7.38 \times 10^{-11} \mathrm{~W} \mathrm{~kg}^{-1}$ (Turcotte and Schubert, 2002).

The local Nusselt number $N u_{r}$ is a measure for the efficiency of radial heat transport in terms of the ratio of total heat flux to conductive heat flux and can be written as

$$
N u_{r}=\left(1+\frac{k_{v}}{k_{c}}\right)\left[1-\frac{k_{v}}{q}\left(\frac{\mathrm{d} T}{\mathrm{~d} r}\right)_{S}\right]^{-1},
$$

where $(\mathrm{d} T / \mathrm{d} r)_{S}=-(\gamma g T) / \Phi$ is the adiabatic temperature gradient. The effective thermal conductivity $k_{v}$ due to convection can be inferred from applying a mixing length formulation. The basic idea is that the heat transfer is primarily due to vertical motion of a fluid parcel. Sasaki and Nakazawa (1986) and Abe (1997) extended this concept for highly viscous fluids by considering that the Stokes' viscous drag is balanced by the buoyancy force operating on such a parcel. Mixing length formulations have been applied in planetary sciences by Senshu et al. (2002) to model the thermal history of early Mars and by Kimura et al. (2009) to investigate the thermal evolution of the Jovian moon Ganymede. Using this approach, $k_{v}$ can be calculated 


$$
k_{v}=\frac{l^{4} \rho^{2} \alpha^{2} g \Phi}{18 \gamma \eta}\left[\left(\frac{\mathrm{d} T}{\mathrm{~d} r}\right)_{S}-\frac{\mathrm{d} T}{\mathrm{~d} r}\right],
$$

where $l$ is the characteristic mixing length, $\alpha$ is the thermal expansivity of the mantle, and $\eta$ is the local dynamic viscosity. Following Abe (1997) and Senshu et al. (2002), we treat the mixing length $l$ as the distance from the nearest boundary of the convective layer. The thermal expansivity is evaluated locally using the definition of the thermodynamic Grüneisen parameter $\gamma=\left(\alpha K_{S}\right) /\left(\rho C_{P}\right)$, where $C_{P}$ is the specific heat capacity of the lower mantle of about $1250 \mathrm{~J} \mathrm{~kg}^{-1} \mathbf{K}^{-1}$.

In the present study, we model a pressure- and temperaturedependent viscosity $\eta$ defined as

$$
\eta=\frac{\sigma}{2 \dot{\epsilon}}
$$

where $\sigma$ is the applied shear stress and $\dot{\epsilon}$ is the shear strain rate. A general flow law for steady state creep can be written as (e.g., Ranalli, 2001)

$$
\dot{\epsilon}=A d^{m} \sigma^{p} \exp \left(-\frac{E^{*}+P V^{*}}{R T}\right),
$$

where $d$ is the grain size, $R$ is the universal gas constant, and $A$, $p, m, E^{*}$, and $V^{*}$ are flow constants related to the dominant creep mechanism. Seismic observations suggest that diffusion $(p=1)$ is the predominant creep mechanism in the lower mantle of the 
Earth, while dislocation $(p \geq 3.5)$ dominates the flow of the Earth's upper mantle (e.g., Karato et al., 1995). Therefore, we assume diffusion-controlled creep $\left(d \sim 10^{-3} \mathrm{~m}\right)$ in the $\mathrm{MgSiO}_{3}$ lower mantle and dislocation-controlled creep at a constant strain rate of $\dot{\epsilon}=$ $10^{-15} \mathrm{~s}^{-1}$ in the $\mathrm{Mg}_{2} \mathrm{SiO}_{4}$ upper mantle. We also assume a dry rheology and use activation parameters experimentally obtained by Karato and Wu (1993). To account for the depth dependence of the activation volume $V^{*}$, we approximate the pressure-induced shrinkage of the activation volume as a vacancy in the material (O'Connell, 1977). A similar approach was recently applied by Fu et al. (2010) to calculate the pressure-dependent activation volume of water-ice polymorphs.

We implicitly solve equations [1]-[3] in conjunction with equations [4]-[6] by numerical integration employing a BDF (backward differentiation formulae or Gear's method) routine. The algorithm starts the radial integration in the center of the model planet $(r=0)$ using central boundary conditions of $m(0)=0, g(0)=0, P(0)=P_{c}, q(0)=0$, and $T(0)=T_{c}$ where $P_{c}$ and $T_{c}$ are educated initial guesses for the central pressure and temperature, respectively. Integration then proceeds outward through each shell until the total mass $M_{p}$ of the planet is achieved. If necessary, this process will start over with iteratively adjusted central pressure $P_{c}$ and temperature $T_{c}$. The algorithm stops integrating if the surface boundary conditions of $m\left(R_{p}\right)=M_{p}$, $P\left(R_{p}\right)=P_{s}$, and $T\left(R_{p}\right)=T_{s}$ are met at $r=R_{p}$.

\subsection{Equation of State}

The local density is calculated by 


$$
\rho(r)=f_{E o S}(P(r), T(r)),
$$

where $f_{E o S}$ is the equation of state (EoS), a unique function relating to each other density, pressure, and temperature of a given material in thermal equilibrium. Almost all EoS are semi-empirical fits to experimental data obtained by high-pressure experiments or seismological observations.

\subsubsection{Isothermal Equation of State}

In the upper silicate mantle we use a third-order Birch-Murnaghan EoS to calculate how the density of a given material behaves with increasing pressure at a constant reference temperature (Birch, 1952):

$$
P=\frac{3}{2} K_{0}\left(x^{7 / 3}-x^{5 / 3}\right)\left[1+\frac{3}{4}\left(K_{0}^{\prime}-4\right)\left(x^{2 / 3}-1\right)\right],
$$

where $x=\rho / \rho_{0}$ is the compression ratio with respect to the ambient density $\rho_{0} ; K_{0}$ and $K_{0}{ }^{\prime}$ denote the isothermal bulk modulus and its pressure derivation at ambient conditions, respectively.

This EoS is based on the expansion of Eulerian finite strain and is widely used in mineralogical and geophysical applications. Almost all high-pressure experiments are fitted to this EoS. However, extrapolation beyond $100 \mathrm{GPa}$ is highly uncertain (Stacey and Davis, 2004). Therefore, we have chosen to implement the following EoS for the lower mantle and the metallic core: (a) the generalized Rydberg EoS (Stacey, 2005), which is derived from the Rydberg interatomic potential function, 
(b) Stacey's reciprocal $K^{\prime}$ EoS (Stacey, 2000), which is directly applicable to seismologically obtained data, and (c) the Keane EoS (Stacey and Davis, 2008) based on the importance of the derivative of the bulk modulus in the limit of infinitely large pressure $K_{\infty}^{\prime}$ (Keane, 1954).

Vinet et al. (1989) proposed a "universal" EoS based on an expression for the cohesive energy that varies only as a function of normalized interatomic separation. This EoS can be written as

$$
P=3 K_{0} x^{\frac{2}{3}}\left(1-x^{-\frac{1}{3}}\right) \exp \left[\frac{2}{3}\left(K_{0}^{\prime}-1\right)\left(1-x^{-\frac{1}{3}}\right)\right]
$$

and is usually called the Vinet EoS. For materials under strong compression, EoS based on an exponential repulsive potential, such as that of Vinet, are superior to finite strain theories (e.g., Hemley et al., 1990; Loubeyre et al., 1996). A comparison of different EoS with theoretically calculated values up to very high pressures $(<1 \mathrm{TPa})$ supports the usage of the Vinet EoS (Hama and Suito, 1996). Later, it was shown by Stacey $(1999,2001)$ that the Vinet EoS is identical to the Rydberg EoS and does not satisfy the thermodynamic requirement of $K_{\infty}^{\prime} \geq \frac{5}{3}$ when extrapolated to infinitely high pressures. Following Stacey (2005), we obtain the generalized Rydberg EoS by adjusting the Vinet equation to arbitrary $K_{\infty}^{\prime}$ by writing

$$
P=3 K_{0} x^{K_{\infty}^{\prime}}\left(1-x^{-\frac{1}{3}}\right) \exp \left[f\left(1-x^{-\frac{1}{3}}\right)\right]
$$

with $f=(3 / 2) K_{0}^{\prime}-3 K_{\infty}^{\prime}+1 / 2$. 


$$
P=K_{0}\left[\frac{K_{0}^{\prime}}{K_{\infty}^{\prime 2}}\left(x^{K_{\infty}^{\prime}}-1\right)-\left(\frac{K_{0}^{\prime}}{K_{\infty}^{\prime}}-1\right) \ln x\right] .
$$

$$
\begin{aligned}
\frac{K}{K_{0}} & =\left(1-K_{\infty}^{\prime} \frac{P}{K}\right)^{-\frac{K_{0}^{\prime}}{K_{\infty}^{\prime}}}, \\
\ln x & =-\left(\frac{K_{0}^{\prime}}{K_{\infty}^{\prime 2}}\right) \ln \left(1-K_{\infty}^{\prime} \frac{P}{K}\right)+\left(1-\frac{K_{0}^{\prime}}{K_{\infty}^{\prime}}\right) \frac{P}{K}
\end{aligned}
$$

The main advantage of equations [16] and [17] is that they can be directly fitted to the Preliminary Reference Earth Model (PREM) (Dziewonski and Anderson, 1981) to obtain the unknown zero pressure parameters $\rho_{0}, K_{0}, K_{0}^{\prime}$, and $K_{\infty}^{\prime}$ for the lower mantle and the core.

Sufficiently similar to the reciprocal $K^{\prime}$ relationship is the Keane EoS

In general, the Keane EoS can be applied more conveniently to laboratory data obtained from high-pressure experiments, whereas 
the reciprocal $K^{\prime}$ EoS is preferred if $\mathbf{P} / \mathbf{K}$ is known from seismic observations. Table 1 lists the parameters $\rho_{0}, K_{0}, K_{0}^{\prime}$, and $K_{\infty}^{\prime}$ used for each material and appropriate EoS.

\subsubsection{Thermal Correction according to Mie-Grüneisen-Debye}

To incorporate the effects of temperature into the EoS, we need to add a thermal pressure term. We use lattice dynamics to take into account temperature effects. In this approach, the crystal is viewed as if composed of a collection of harmonic oscillators. The Helmholtz free energy can then be obtained by summing over all normal mode vibrational frequencies at a given volume. The thermal pressure $\Delta P_{t h}$ is determined by using the Mie-Grüneisen equation (e.g., Jackson and Rigden, 1996):

$$
\Delta P_{t h}(\rho, T)=\gamma \rho\left[E_{t h}(\rho, T)-E_{t h}\left(\rho, T_{0}\right)\right]
$$

where the subscript 0 represents a reference state which is chosen to be the $300 \mathrm{~K}$ isotherm, $\gamma$ is the Grüneisen parameter, and $E_{t h}$ the internal thermal energy.

A simple but successful method to characterize the lattice vibrational modes is the Debye model which treats the solid as a continuous medium and parameterizes the vibrational spectrum in terms of a single characteristic temperature. On that basis, the internal thermal energy $E_{t h}$ at a given temperature can be calculated as

$$
E_{t h}=9 n k_{B} N_{A} T\left(\frac{T}{\theta_{D}}\right)^{3} \int_{0}^{\theta_{D} / T} \frac{z^{3}}{\exp (z)-1} \mathbf{d} z
$$


where $n$ is the number of atoms in the formula unit of the corresponding material, $k_{B}$ is the Boltzmann constant, $N_{A}$ is the Avogadro constant, and $\theta_{D}$ is the Debye temperature.

The Grüneisen parameter $\gamma$ and the Debye temperature $\theta_{D}$ are assumed to be functions of density only. Their values are calculated for the upper silicate mantle as $\gamma=\gamma_{0} x^{-\lambda}$ and $\theta_{D}=\theta_{0} \exp \left[\left(\gamma_{0}-\gamma\right) / \lambda\right]$. The logarithmic volume derivative of the thermodynamic Grüneisen parameter $\lambda$ is taken constant at a value of 3.2 for the upper olivine mantle (Katsura et al., 2009). Within the lower mantle and the metallic core, we use a formulation according to Al'tshuler et al. (1987) for the Grüneisen parameter $\gamma=\gamma_{\infty}+\left(\gamma_{0}-\gamma_{\infty}\right) x^{-\beta}$ and for the Debye temperature $\theta_{D}=\theta_{0} x^{\gamma_{\infty}} \exp \left[\left(1-x^{-\beta}\right)\left(\gamma_{0}-\gamma_{\infty}\right) / \beta\right]$, respectively. The latter formulations describe the theoretically predicted material behavior under high pressure better. Table 2 summarizes the material parameters used for the thermal pressure correction.

For the pure iron core, additional terms are considered to account for electronic and anharmonic thermal pressure $P_{e l}$ and $P_{a n h}$, respectively.

$$
\begin{aligned}
P_{e l}(\rho, T) & =\frac{3}{2} g^{*} n k_{B} N_{A} \rho e_{0} x^{-g^{*}} T^{2}, \\
P_{a n h}(\rho, T) & =\frac{3}{2} m^{*} n k_{B} N_{A} \rho a_{0} x^{-m^{*}} T^{2} .
\end{aligned}
$$

The parameters $e_{0}, g^{*}, a_{0}$, and $m^{*}$ have been obtained by fitting ab initio electronic and anharmonic thermal pressures taken from Dewaele et al. (2006). For example, at a pressure of $300 \mathrm{GPa}$ and a temperature of $6000 \mathrm{~K}$, contributions from electronic and anharmonic pressure for pure iron are 15 
and $3 \mathrm{GPa}$, respectively.

\section{Results}

Using the model approach above, we have computed interior structure models of low-mass, terrestrial-type exoplanets of variable composition.

\subsection{Interior Structure Models}

Figure 1 and 2 show the calculated interior structure for 1, 5, and $10 \mathbf{M}_{\oplus}$ exoplanets with Earth-like bulk composition. Compared are the generalized Rydberg, the Keane, and the reciprocal $K^{\prime}$ EoS. To verify the model approach, we have chosen the Preliminary Reference Earth Model (PREM) (Dziewonski and Anderson, 1981), while a reference geotherm proposed by Stacey and Davis (2008) is used to characterize the present thermal state of the Earth.

\subsubsection{Earth Reference Comparison}

Panel (a)-(c) of Fig. 1 illustrates the density distribution, gravitational acceleration, and hydrostatic pressure as a function of radial distance from the planet's center. A comparison between the three Earth-sized models $\left(1 \mathrm{M}_{\oplus}\right)$ and PREM shows relatively good agreement and therefore validates our model approach. The total mismatch of the calculated planetary radius to PREM is below $0.5 \%$ for all EoS used.

Turning to panel (a) in particular, the core density discrepancy between PREM and the $1 \mathrm{M}_{\oplus}$ models using the generalized Rydberg and the Keane EoS is due to the presence of a lighter element 

324 (b) and (c), respectively.

than iron in the Earth's core. Therefore, the core radius is about $140 \mathrm{~km}$ smaller in these models if compared to the seismologically observed core size. For the $1 \mathrm{M}_{\oplus}$ model using the reciprocal $K^{\prime}$ EoS, the core density discrepancy in comparison to PREM is due to the EoS parameters which are obtained by fitting a "solidified" Earth's outer core. As seen in panel (a), relative to PREM the model predicts slightly lower densities for the inner and somewhat higher densities for the Earth's outer core, whereas the predicted core size is close to that given by PREM.

The density distribution calculated within the mantle is in excellent agreement between the models and PREM. Only a small discrepancy is observed for the lowermost mantle using the EoS fitted to laboratory compression data. This offset is mainly attributed to the addition of magnesiowüstite in the Earth's lower mantle that is not accounted for in the models. As a minor constituent, we expect it to have only a negligible effect on the interior structure of massive terrestrial exoplanets. However, due to a relatively high thermal conductivity, it may have an impact on the efficiency of the heat transport and thereby might affect the mantle convection pattern. A relatively small discrepancy is observed in the so-called transition region of the Earth's upper mantle where consecutive phase transitions are occurring, since those are not implemented in all three model calculations for the sake of simplicity. Similar observations are valid for the radial distribution of gravitational acceleration and hydrostatic pressure as illustrated in panel

In panel (d) of Fig. 1 the radial temperature distributions calcu- 
lated for the generalized Rydberg, the Keane, and the reciprocal $K^{\prime}$ model are compared to the reference geotherm. The temperature profiles of the Earth-sized models are in good agreement with the latter. For all three models, the calculated upper mantle geotherm is slightly colder when compared to the reference. This difference can be attributed to the simplifying assumption of an upper mantle composed of olivine, thereby neglecting pressure-induced phase transitions to $\beta$ and $\gamma$ spinel at shallow depth. The discrepancy of the temperature distribution within the lower mantle is attributed to principal uncertainties of the calculated radial viscosity profile governed by creep activation parameters and the actual grain size distribution of lower mantle minerals. Overall, the comparison between the calculated temperature profiles of the $1 \mathrm{M}_{\oplus}$ models and a reference geotherm indicates that the mixing length approach is consistent with other estimates.

\subsubsection{EoS Comparison}

The density as a function of radial distance from the planet's center is shown in Fig. 2 panel (a). In all models of 5 and $10 \mathrm{M}_{\oplus}$, the density increases almost linearly within the dominant mantle regions composed of olivine, perovskite, and post-perovskite, respectively. A pronounced density variation of about $700 \mathrm{~kg} \mathrm{~m}^{-3}$ occurs at the phase transition boundary between the upper olivine mantle and the underlying perovskite layer. Due to similar elastic properties of perovskite and post-perovskite, the phase transition from the intervening perovskite layer to the lowermost post-perovskite mantle is hardly visible. For the models using the generalized Rydberg EoS, density 
variations ranging from $8090\left(5 \mathrm{M}_{\oplus}\right)$ to $9430 \mathrm{~kg} \mathrm{~m}^{-3}\left(10 \mathrm{M}_{\oplus}\right)$ are found at the core-mantle boundary, which represents a chemical material transition from silicate to iron. In comparison, the models using the Keane EoS produce larger density variations at the core-mantle boundary ranging from $8560\left(5 \mathrm{M}_{\oplus}\right)$ to $10,500 \mathrm{~kg} \mathrm{~m}^{-3}$ $\left(10 \mathbf{M}_{\oplus}\right)$. Smaller density variations ranging from $6700\left(5 \mathbf{M}_{\oplus}\right)$ to $8000 \mathrm{~kg} \mathrm{~m}^{-3}\left(10 \mathbf{M}_{\oplus}\right)$ are observed for the models using the reciprocal $K^{\prime}$ EoS. Within the iron cores, the density increases parabolically with depth from the core-mantle boundary by about $30 \%$ to reach central densities of 20,600 and $24,900 \mathrm{~kg} \mathrm{~m}^{-3}$ for the models using the generalized Rydberg EoS and by about $40 \%$ to central densities of 23,400 and $30,100 \mathrm{~kg} \mathrm{~m}^{-3}$ for the Keane models, respectively. For the models using the reciprocal $K^{\prime} \mathrm{EoS}$, the density increase amounts to about $35 \%$, reaching central density values of 20,400 and $25,800 \mathrm{~kg} \mathrm{~m}^{-3}$.

Upon comparison of core density distributions predicted by the different models, it is obvious that the difference in core densities becomes much larger for more massive model planets. This is related to (a) the extrapolation of different EoS and (b) the corresponding increasingly pronounced density discrepancy within the lower mantle. It is seen that the predicted central density for the 1 to $10 \mathrm{M}_{\oplus}$ exoplanets increases tenfold from about 2 to $20 \%$ comparing the generalized Rydberg to the Keane EoS. This corresponds to a core size uncertainty in the range of $5 \%$ for the most massive Earth-like exoplanets. 
The difference in mantle density at the core-mantle boundary increases from almost zero for the $1 \mathrm{M}_{\oplus}$ models to slightly more than $12 \%$ in the case of the $10 \mathbf{M}_{\oplus}$ planets when comparing the models using the generalized Rydberg to those using the Keane EoS. A similar comparison between the generalized Rydberg and the reciprocal $K^{\prime}$ EoS models yield a maximum density discrepancy at the core-mantle boundary of about $15 \%$. This can be attributed to (a) the extrapolation of different EoS and (b) the method how the material parameters for both the generalized Rydberg and the reciprocal $K^{\prime}$ model are obtained. The reciprocal $K^{\prime}$ EoS represents a fit to the Earth's lower mantle and, therefore, accounts for only a small amount of post-perovskite within the lower mantle. Hence, the mantles of model planets calculated by applying the reciprocal $K^{\prime}$ EoS are predominantly composed of perovskite.

Since post-perovskite is expected to be the predominant mineral phase within terrestrial-type exoplanets, the perovskite to postperovskite phase transition is explicitly incorporated in the models using the generalized Rydberg and the Keane EoS. For example, for a planet ten times as massive as the Earth, our calculations indicate that about $43 \%$ of the planet's radial extent would consist of post-perovskite. Under this assumption, the models using fits to laboratory data are more suitable to predict the deep mantle structure of low-mass exoplanets, because the reciprocal $K^{\prime}$ EoS does not account for the material parameter change from perovskite to the post-perovskite phase. Nevertheless, we find that the predicted planetary radius 
for an $10 \mathbf{M}_{\oplus}$ Earth-like exoplanet merely differs by less than $2 \%$ between all three EoS, thereby constraining the maximum uncertainty due to the implemented EoS and compositional differences.

For the gravitational acceleration shown in panel (b) of Fig. 2, we observe a linear increase for all planets from the center to the core-mantle boundary, where peak values are attained. The subsequent parabolic decrease merges into a roughly constant gravitational acceleration throughout the mantle. The average surface gravity is found to be $20.0\left(5 \mathrm{M}_{\oplus}\right)$ and $27.7 \mathrm{~m} \mathrm{~s}^{-2}$ $\left(10 \mathbf{M}_{\oplus}\right)$ for the models using the generalized Rydberg EoS, 20.4 $\left(5 \mathbf{M}_{\oplus}\right)$ and $28.9 \mathbf{m ~ s}^{-2}\left(10 \mathbf{M}_{\oplus}\right)$ for the models using the Keane EoS, and $20.5\left(5 \mathbf{M}_{\oplus}\right)$ and $29.2 \mathbf{m ~ s}^{-2}\left(10 \mathbf{M}_{\oplus}\right)$ for those using the reciprocal $K^{\prime}$ EoS, respectively.

In panel (c) of Fig. 2 variations with radial distance from the planet's center of the hydrostatic pressure are compared for Earth-like exoplanets of 5 and $10 \mathrm{M}_{\oplus}$, respectively. A linear pressure increase through the mantle is observed in all models. For the models using the generalized Rydberg EoS, pressures of 645 and $1290 \mathrm{GPa}$ are attained at the core-mantle boundary. In comparison, for the models using the Keane and the reciprocal $K^{\prime}$ EoS we find higher pressures of 701, 1500 and $670,1430 \mathrm{GPa}$ at the core-mantle boundary, respectively. Within the core, a parabolical increase of pressure with depth occurs in all models. For the $5 \mathbf{M}_{\oplus}$ planets, the central pressure is found to be 1920, 2170, and 1910 GPa using the generalized Rydberg, the Keane, and the reciprocal $K^{\prime}$ EoS, respectively. For the $10 \mathbf{M}_{\oplus}$ planets, the corresponding central pressures are 3870, 4110, and 4750 GPa. 
Upon comparison of the resulting radial pressure distributions using generalized Ryberg and Keane EoS, we find that the Keane EoS predicts systematically higher pressures in the interior of massive exoplanets. An increasingly pronounced central pressure discrepancy is attaining about $20 \%$ for the $10 \mathbf{M}_{\oplus}$ planets. This illustrates how differently both EoS extrapolate to the high-pressure limit. A similar comparison between the central pressures calculated according to generalized Rydberg and reciprocal $K^{\prime}$ EoS yields an appreciable smaller pressure discrepancy reflecting the less dense iron alloy core represented by the reciprocal $K^{\prime}$ models. As shown in panel (c), both the central pressure and the pressure at the core-mantle boundary of an Earth-like exoplanets scale linearly with total mass.

Panel (d) of Fig. 2 illustrates the radial distribution of temperature for Earth-like exoplanets of 5 and $10 \mathrm{M}_{\oplus}$, respectively. The temperature increases rapidly from an arbitrarily fixed surface value of 300 to about $1400 \mathrm{~K}$ within the topmost part of the upper mantle as a result of the mixing length approach. In this region, heat is predominantly transferred by conduction, followed by an adiabatic temperature rise across the convecting mantle. We observe an increasingly super-adiabatic temperature rise toward the core-mantle boundary. This is interpreted in terms of a sluggish convective regime within the lowermost mantle of more massive planets. For the models using the generalized Rydberg EoS, the temperature at the core-mantle boundary is found to be $5510 \mathrm{~K}$ for $5 \mathrm{M}_{\oplus}$ and $6650 \mathrm{~K}$ for $10 \mathbf{M}_{\oplus}$. The corresponding central temperatures are 7970 and 
$9790 \mathrm{~K}$, respectively. Using the Keane EoS, the temperature at the core-mantle boundary reaches 5770 and $7280 \mathrm{~K}$, whereas temperatures of 9080 and $11,800 \mathbf{K}$ would prevail in the center of 5 and $10 \mathbf{M}_{\oplus}$ planets, respectively. Applying the reciprocal $K^{\prime}$ EoS yields temperatures at the core-mantle boundary of 6470 and $8190 \mathrm{~K}$ and corresponding central temperatures of 9730 and $12,600 \mathrm{~K}$, respectively. These results differ from those obtained by using parameterized convection models, which in general yield substantially colder interiors (Valencia et al., 2006). Compared to Valencia et al. (2006), our results yield a mean temperature value at the core-mantle boundary in excess of $1900\left(5 \mathbf{M}_{\oplus}\right)$ and $2500 \mathbf{K}\left(10 \mathbf{M}_{\oplus}\right)$, respectively. In accordance with previous studies (e.g., Seager et al., 2007), the pronounced differences in core temperature do not much affect the overall structure of the corresponding planetary interiors as shown in Fig. 2.

\subsubsection{Mantle viscosity comparison}

The radial temperature distribution is mainly controlled by the mantle viscosity, which is treated in form of a temperature- and pressure dependent Arrhenius law. Figure 3 panel (a) shows the calculated mantle viscosity for 1,5 , and $10 \mathbf{M}_{\oplus}$ exoplanets with Earth-like bulk composition using the generalized Rydberg models for a case study. It can be seen that the $1 \mathrm{M}_{\oplus}$ model has the largest viscosity contrast within the mantle and the highest peak viscosity $\left(\sim 5 \times 10^{23} \mathrm{~Pa}\right.$ s) when compared to more massive exoplanets. Large viscosity variations are situated in the upper and the lowermost part of all mantles and can be attributed to 
thermal boundary layers. Within the $1 \mathbf{M}_{\oplus}$ model, we observe the lowest viscosity at the core-mantle boundary, whereas for massive exoplanets the lowest viscosity appears in the upper mantle and higher values are found at the core-mantle boundary due to a more sluggish convective regime. Despite the increasing pressure within more massive exoplanets, mantle viscosities stay relatively constant over an extended mantle region due to the decrease in activation volume and increase of temperature with depth. The viscosity-controlling feedback mechanism involving temperature, pressure, and activation volume seems closely related to the Tozer effect (e.g., Tozer, 1972) as observed on Earth. A detailed analysis of this effect, however, would involve the calculation of thermal history of massive exoplanets.

\subsection{Mass-radius Relations and Scaling Laws}

Using our model approach, we investigate different types of low-mass exoplanets, characterized by variable bulk compositions. We apply the generalized Rydberg EoS and calculate the planetary radius as a function of mass for internally differentiated Earth-like, Mercury-type, and ocean planets. Figure 4 shows the resulting mass-radius relations for several classes of low-mass exoplanets (dashed lines). Furthermore, the red line in Fig. 4 resembles the relation between mass and radius of a self-compressible, pure silicate sphere. It divides fully differentiated dry planets from those containing a certain amount of volatiles. The curves indicating pure $\mathrm{H}_{2} \mathrm{O}$ and iron spheres confine the possible range of mass-radius relations of Earth-like and ocean planets. Planets less dense than a pure water-ice sphere must have a 
significant gas envelope and planets denser than a pure iron sphere are unlikely to exist (Seager et al., 2007). Also included in Fig. 4 is the position of the Earth, which perfectly fits the mass-radius curve for Earth-like planets.

Moreover, we show the relative position of the CoRoT-7b exoplanet within its observational uncertainties. The nature of CoRoT7b has been intensely discussed due to a wide range of mass estimates taken from the literature. The discovering paper of Léger et al. (2009) published a planetary radius of $(1.68 \pm 0.09) \mathbf{R}_{\oplus}$ which was obtained by analyzing CoRoT lightcurves. Complementing this study, Queloz et al. (2009) reported the first mass estimate for CoRoT-7b of $(4.8 \pm 0.8) \mathbf{M}_{\oplus}$ by employing the High Accuracy Radial velocity Planet Searcher (HARPS). Later, Bruntt et al. (2010) evaluated the same data sets and revised the planets radius to $(1.58 \pm 0.10) \mathbf{R}_{\oplus}$ and its total mass to $(5.2 \pm 0.8) \mathbf{M}_{\oplus}$. Hatzes et al. (2010) and Ferraz-Mello et al. (2010), taking into account the possible presence of more than two planets orbiting CoRoT-7, obtain systematically higher masses of $(6.9 \pm 1.4) \mathbf{M}_{\oplus}$ and $(8.0 \pm 1.2) \mathbf{M}_{\oplus}$, respectively. Using star spot modeling techniques, Boisse et al. (2011) favor a planetary mass of $(5.7 \pm 2.5) \mathrm{M}_{\oplus}$ for CoRoT-7b. Pont et al. (2010) argue that CoRoT-7b may possess a relatively low mass in the order of $1-4 \mathbf{M}_{\oplus}$ and even questioning its existence. Hatzes et al. (2011) have revisited previous mass estimates and suggest a planetary mass of $(7.38 \pm 0.34) \mathbf{M}_{\oplus}$ as currently most reliable estimate for CoRoT-7b. Applying the mass-radius relationships shown in Fig. 4, we find that CoRoT-7b is predominantly com- 
posed of rock and iron, with an iron content ranging from Earthlike (67.5 wt.-\% silicate +32.5 wt.-\% iron) to Mercury-like (30 wt.$\%$ silicate +70 wt.- $\%$ iron). Furthermore, we have added other recently discovered transiting low-mass exoplanets such as Kepler10b (Batalha et al., 2011) that is similar to CoRoT-7b in terms of bulk composition. Contrary, GJ 1214b (Charbonneau et al., 2009), Kepler-11b, and Kepler-11f (Lissauer et al., 2011) rather resemble hot gasous planets due to their low average densities.

Finally, we perform a power law fit of $R / R_{\oplus} \propto\left(M / M_{\oplus}\right)^{\beta}$ to the calculated mass-radius relations and obtain scaling exponents for the three classes of low-mass exoplanets. Table 3 summarizes the obtained scaling exponents $\beta$ for the considered low-mass exoplanets valid for a mass range from one to ten times the mass of the Earth. These are in good agreement with scaling laws previously proposed by Valencia et al, (2007a) and Sotin et al. (2007), thereby underscoring the validity of our model approach.

\section{Discussion and Conclusions}

This study focuses on the comparison of different equations of state (EoS). The models using the generalized Rydberg and the Keane EoS are consistent with most recent findings obtained from ab initio calculations. Hence, the main advantage is that they account for a post-perovskite phase transformation in the lowermost mantle. Recent ab inito calculations imply that this mineralogical phase of $\mathrm{MgSiO}_{3}$ should be stable up to $1 \mathrm{TPa}$, but its material parameters are subject to discussion and, in comparison 
to perovskite, less well constrained due to the current limitations of high-pressure experiments. In contrast, the model using the reciprocal $K^{\prime}$ EoS essentially scales up the present Earth based on seismological evidence, thereby representing a truly Earth-like composition. The main advantage of that approach is that neither high-pressure experiments nor ab initio calculations are involved to a larger extent; the EoS parameters are essentially fitted to the Earth as primary reference.

A major uncertainty in modeling the interior structure of low-mass exoplanets arises due to the lack of experimental data to reliably parameterize the EoS in the high-pressure range from 200 up to 10,000 GPa (Seager et al., 2007; Grasset et al., 2009). To address this problem, we have used only EoS that are compliant with the thermodynamics of the high-pressure limit to investigate implications on planetary mass and radius. Although neither a necessary nor a sufficient condition for an accurate EoS implementation at a given pressure range, the latter can be used to impose an additional constraint for meaningful extrapolation to high pressures in the absence of experimental and theoretical data. This characteristics makes the extrapolation not only consistent with the physics of thermodynamic limits but also more trustable. It should be pointed out, however, that due to the limitation of extrapolation itselves no model used so far is capable to account for possible mineralogical phase transitions which may occur beyond the post-perovskite phase. Therefore, the extrapolation approach yields only a lower bound of the density distribution within planets more massive than the Earth. 
As expected, the reciprocal $K^{\prime}$ EoS yields an almost perfect fit to PREM since the corresponding fit parameters are obtained from a wealth of seismological observations. For planets more massive than the Earth, however, the generalized Rydberg and the Keane EoS are found to be more suitable since the material parameters of post-perovskite are explicitly taken into account. Post-perovskite is expected to be the predominant mineral phase assemblage present within the interiors of lowmass Earth-like exoplanets. Despite the fact that different methods were applied to fit these EoS to laboratory data or seismological observations, respectively, the resultant total planetary radii only differ by a relatively small amount for low-mass Earth-like exoplanets.

To calculate the radial temperature structure within the model planets we have applied a mixing length formulation instead of parameterized convection models previously used by Valencia et al. (2006). Contrary to the latter, the mixing length method uses local parameters to describe the efficiency of the heat transport instead of allocating a global Rayleigh number to convective mantle layers. This approach allows to self-consistently calculate radial temperature profiles of massive exoplanets. Whereas Sotin et al. (2007) used fixed temperature variations based on Earth-like values at boundary layers, Seager et al. (2007) treated those as second-order effects and entirely neglect the influence of temperature for planets more massive than the Earth. Compared to the forementioned studies, the models presented in this study result in generally hotter planets, primarily due to a steeper temperature gradient prevalent in the deep interior that is mainly attributed to the pressure-induced increase of 
viscosity at elevated lower mantle pressures. As a consequence, planets more massive than the Earth will experience an increasing tendency to extended sluggish convective regimes in the lowermost mantle because of the reduced efficiency of convective heat transport with increasing mantle pressures.

Furthermore, a possible metallization of some oxides at ultrahigh pressures could result in less efficient radiative heat transfer (e.g., Umemoto et al., 2006) and generally reduced mantle viscosities (Karato, 2011), thereby affecting the thermal state of the lowermost mantle of Earth-like exoplanets. Here, we considered that post-perovskite remains an electrical insulator and metallization does not occur within the investigated pressure range up to $1.5 \mathrm{TPa}$. This is supported by recent experiments of an $\mathrm{MgSiO}_{3}$ analog implying that the dissociation of $\mathrm{MgSiO}_{3}$ may occur at higher pressures than previously predicted (Grocholski et al., 2010). Ab initio calculations suggest that at least $\mathrm{MgO}$ remains insulating before metallizing at ultra-high pressures of about 21 TPa (Oganov et al., 2003). For the metallic core, we have added terms to account for the electronic and anharmonic thermal pressure in accordance with ab inito calculations. Especially the thermal excitation of electrons of metals like iron cannot be neglected, because it amounts to about $20 \%$ of the total thermal pressure under Earth core conditions.

In the present study, scaling coefficients are obtained which are in good agreement with those proposed previously for low-mass exoplanets. The different EoS used, the slightly different bulk compositions assumed, and the different approaches to model radial temperature profiles are the main reason 
for the small discrepancies seen in the scaling coefficients. This similarity is mainly attributed to the general insensitivity of mass-radius relations to the present thermal state of planetary interiors as a consequence of the increasingly close-packed matter due to elevated pressures. This finding is in agreement with previous models by Sotin et al. (2007) and Seager et al. (2007), thereby indicating the robustness of mass-radius relations and their usage for the classification of extrasolar planets in terms of bulk composition.

According to the predictions of planet formation models and observational surveys, low-mass exoplanets beyond the solar system should be quite abundant (e.g., Howard et al., 2010). The discovery of terrestrial-type exoplanets relies to a large extent on current detection limits of ground-based observational methods. For example, existing space-based telescopes like CoRoT or Kepler, and future space missions under study such as PLATO, are even capable to precisely measure the radius of relatively small planets transiting their host stars. Complementary to this technique, precise radial velocity observations provide the corresponding planetary mass. Our model calculations using different EoS for low-mass exoplanets with fixed bulk composition indicate that the uncertainty in calculated planetary radius will be substantially smaller than typical measurement uncertainties from transit photometry. Moreover, Fig. 4 clarifies that planetary mass and radius impose equally important constraints on model planets as massive as the Earth, whereas in the upper mass range interior structure models are chiefly constrained by precise determinations of planetary radius. It also illustrates that it is more difficult to distinguish a Mercury-like from an Earth-like planet 
than an Earth-like from an ocean planet. Furthermore, if cosmochemical arguments are taken into account, it is possible to reduce or eliminate principal non-uniquenesses in terms of bulk composition. For example, the close proximity of CoRoT-7b and Kepler-10b to their host stars may suggest that water-ice as a degenerative constituent would be only minor, or even absent. To interrelate this, for planetary objects more massive than $10 \mathrm{M}_{\oplus}$, typical ground-based surveys with given measurement uncertainties of $\pm 10 \%$ would suffice to readily distinguish between the three principal classes of low-mass exoplanets. In the intermediate mass range from 5 to $10 \mathrm{M}_{\oplus}$, space telescopes like CoRoT and Kepler with observational uncertainties of $\pm 5 \%$ are well suited to deliver radius measurements precisely enough to distinguish between terrestrial-type and ocean planets. For Earth-sized exoplanets, however, new space missions such as, e.g., PLATO are needed in combination with equally precise mass determinations to reliably deduce the bulk composition for classification purposes.

\section{Acknowledgments}

We are grateful to the anonymous reviewers for their constructive comments and valuable suggestions that considerably improved the manuscript. This research has been supported by the Helmholtz Association through the research alliance "Planetary Evolution and Life". 
671

672

673

0

\section{References}

Abe, Y., 1997. Thermal and chemical evolution of the terrestrial magma ocean. Phys. Earth Planet. Inter. 100, 27-39.

Al'tshuler, L. V., Brusnikin, S. E., Kuz'menkov, E. A., 1987. Isotherms and Grüneisen functions for 25 metals. J. Appl. Mech. Tech. Phys. 28, 129-141.

Batalha, N. M., Borucki, W. J., Bryson, S. T., Buchhave, L. A., Caldwell, D. A., et al., 2011. Kepler's first rocky planet: Kepler-10b. Astrophys. J. 729 .

Birch, F., 1952. Elasticity and constitution of the Earth's interior. J. Geophys. Res. 57, 227-286.

Boisse, I., Bouchy, F., Hebrard, G., Bonfils, X., Santos, N., Vauclair, S., 2011. Disentangling between stellar activity and planetary signals. Astron. \& Astrophys. 528, A4.

Bruntt, H., Deleuil, M., Fridlund, M., Alonso, R., Bouchy, F., et al., 2010. Improved stellar parameters of CoRoT-7: A star hosting two super-Earths. Astron. \& Astrophys. 519, A51.

Charbonneau, D., Berta, Z. K., Irwin, J., Burke, C. J., Nutzman, P., et al., 2009. A super-Earth transiting a nearby low-mass star. Nature 462, 891894.

Dewaele, A., Loubeyre, P., Occelli, F., Mezouar, M., Dorogokupets, P. I., Torrent, M., 2006. Quasihydrostatic equation of state of iron above 2 Mbar. Phys. Rev. Lett. 97, 215504. 
714 Grocholski, B., Shim, S. H., Prakapenka, V. B., 2010. Stability of the $\mathrm{MgSiO}_{3}$

Dubrovinsky, L. S., Saxena, S. K., Dubrovinskaia, N. A., Rekhi, S., Bihan, T. L., 2000. Grüneisen parameter of $\epsilon$-iron up to $300 \mathrm{GPa}$ from in-situ X-ray study. Am. Mineral. 85, 386-389.

Dziewonski, A. M., Anderson, D. L., 1981. Preliminary reference Earth model. Phys. Earth Planet. Inter. 25, 297-356.

Fei, Y., Orman, J. V., Li, J., van Westrenen, W., Sanloup, C., et al., 2004. Experimentally determined postspinel transformation boundary in $\mathrm{Mg}_{2} \mathrm{SiO}_{4}$ using $\mathrm{MgO}$ as an internal pressure standard and its geophysical implications. J. Geophys. Res. 109, B02305.

Ferraz-Mello, S., dos Santos, M. T., Beauge, C., Michtchenko, T. A., Rodriguez, A., 2010. On planetary mass determination in the case of superEarths orbiting active stars. The case of the CoRoT-7 system. Astron. \& Astrophys., accepted.

Fortney, J. J., Marley, M. S., Barnes, J. W., 2007. Planetary radii across five orders of magnitude in mass and stellar insolation: Application to transits. Astrophys. J. 659, 1661-1672.

Fu, R., O'Connell, R. J., Sasselov, D. D., 2010. The interior dynamics of water planets. Astrophys. J. 708, 1326-1334.

Grasset, O., Schneider, J., Sotin, C., 2009. A study of the accuracy of massradius relationships for silicate-rich and ice-rich planets up to 100 Earth masses. Astrophys. J. 693, 722-733. 
analog $\mathrm{NaMgF}_{3}$ and its implication for mantle structure in super-Earths. Geophys. Res. Lett. 37, L14204.

Hama, J., Suito, K., 1996. The search for a universal equation of state correct up to very high pressures. J. Phys.: Condens. Matter 8, 67-81.

Hatzes, A. P., Dvorak, R., Wuchterl, G., Guterman, P., Hartmann, M., et al., 2010. An investigation into the radial velocity variations of CoRoT-7. Astron. \& Astrophys. 520, A93.

Hatzes, A. P., Fridlund, M., Carone, L., Pätzold, M., Valencia, D., et al., 2011. On the mass of CoRoT-7b, in preparation.

Hemley, R. J., Mao, H. K., Finger, L. W., Jephcoat, A. P., Hazen, R. M., Zha, C. S., 1990. Equation of state of solid hydrogen and deuterium from single-crystal X-ray diffraction to 26.5 GPa. Phys. Rev. B 42, 6458-6470.

Hernlund, J. W., Labrosse, S., 2007. Geophysically consistent values of the perovskite to post-perovskite transition Clapeyron slope. Geophys. Res. Lett. 34, L05309.

Hofmeister, A. M., 1999. Mantle values of thermal conductivity and the geotherm from phonon lifetimes. Science 283, 1699-1706.

Howard, A. W., Marcy, G. W., Johnson, J. A., Fischer, D. A., Wright, J. T., et al., 2010. The occurrence and mass distribution of close-in super-Earths, Neptunes, and Jupiters. Science 330, 653-655.

Isaak, D. G., Anderson, O. L., 2003. Thermal expansivity of HCP iron at very high pressure and temperature. Physica B 328, 345-354. 
Jackson, I., Rigden, S. M., 1996. Analysis of P-V-T data: Constraints on the thermoelastic properties of high-pressure minerals. Phys. Earth Planet. Inter. $96,85-112$.

Karato, S., 2011. Rheological structure of the mantle of a super-Earth: Some insights from mineral physics. Icarus 212, 14-23.

Karato, S., Zhang, S., Wenk, H. R., 1995. Superplasticity in Earth's lower mantle: Evidence from seismic anisotropy and rock physics. Science 270, $458-461$.

Karato, S.-I., Wu, P., 1993. Rheology of the upper mantle: A synthesis. Science 260, 771-778.

Katsura, T., Shatskiy, A., Manthilake, M. A. G. M., Zhai, S., Fukui, H., et al., 2009. Thermal expansion of forsterite at high pressures determined by in-situ X-ray diffraction: The adiabatic geotherm in the upper mantle. Phys. Earth Planet. Inter. 174, 86-92.

Keane, A., 1954. An investigation of finite strain in an isotropic material subjected to hydrostatic pressure and its seismological applications. Aust. J. Phys. 7, 322-333.

Kimura, J., Nakagawa, T., Kurita, K., 2009. Size and compositional constraints of Ganymede's metallic core for driving an active dynamo. Icarus $202,216-224$.

Kuskov, O. L., Kronrod, V. A., 2001. Core sizes and internal structure of Earth's and Jupiter's satellites. Icarus 151, 204-227. 
Léger, A., Rouan, D., Schneider, J., Barge, P., Fridlund, M., et al., 2009. Transiting exoplanets from the CoRoT space mission. VIII. CoRoT-7b: The first super-Earth with measured radius. Astron. \& Astrophys. 506, 287-302.

Léger, A., Selsis, F., Sotin, C., Guillot, T., Despois, D., et al., 2004. A new family of planets?"Ocean-planets". Icarus 169, 499-504.

Lissauer, J. J., Fabrycky, D. C., Ford, E. B., Borucki, W. J., Fressin, F., et al., 2011. A closely packed system of low-mass, low-density planets transiting Kepler-11. Nature 470, 53-58.

Loubeyre, P., LeToullec, R., Hausermann, D., Hanfland, M., Hemley, R. J., et al., 1996. X-ray diffraction and equation of state of hydrogen at megabar pressures. Nature 383, 702-704.

Loubeyre, P., LeToullec, R., Wolanin, E., Hanfland, M., Hausermann, D., 1999. Modulated phases and proton centring in ice observed by X-ray diffraction up to 170 GPa. Nature 397, 503-506.

Mayor, M., Bonfils, X., Forveille, T., Delfosse, X., Udry, S., et al., 2009. The HARPS search for southern extra-solar planets? XVIII. An Earthmass planet in the GJ 581 planetary system. Astron. \& Astrophys. 507, $487-494$.

O'Connell, R. J., 1977. On the scale of mantle convection. Tectonophysics $38,119-136$.

Oganov, A. R., Brodholt, J. P., Price, G. D., 2001. The elastic constants of 
$\mathrm{MgSiO}_{3}$ perovskite at pressures and temperatures of the Earth's mantle. Nature 411, 934-937.

Oganov, A. R., Gillan, M. J., Price, G. D., 2003. Ab initio lattice dynamics and structural stability of MgO. J. Chem. Phys. 118, 10174-10182.

Oganov, A. R., Ono, S., 2004. Theoretical and experimental evidence for a post-perovskite phase of $\mathrm{MgSiO}_{3}$ in Earth's D" layer. Nature 430, 445-448.

Ono, S., Oganov, A. R., 2005. In-situ observations of phase transition between perovskite and $\mathrm{CaIrO}_{3}$-type phase in $\mathrm{MgSiO}_{3}$ and pyrolitic mantle composition. Earth Planet. Sci. Lett. 236, 914-932.

Pont, F., Aigrain, S., Zucker, S., 2010. Reassessing the radial-velocity evidence for planets around CoRoT-7. Mon. Not. R. Astron. Soc. 411, 19531962.

Queloz, D., Bouchy, F., Moutou, C., Hatzes, A., Hebrard, G., et al., 2009. The CoRoT-7 planetary system: Two orbiting super-Earths. Astron. \& Astrophys. 506, 303-319.

Ranalli, G., 2001. Mantle rheology: radial and lateral viscosity variations inferred from microphysical creep laws. J. Geodyn. 32, 425-444.

Rogers, L., Seager, S., 2010. A framework for quantifying the degeneracies of exoplanet interior compositions. Astrophys. J. 712, 974-991.

Sasaki, S., Nakazawa, K., 1986. Metal-silicate fractionation in the growing Earth - Energy source for the terrestrial magma ocean. J. Geophys. Res. 91, 9231-9238. 
Seager, S., Kuchner, M., Hier-Majumder, C. A., Militzer, B., 2007. Massradius relationships for solid exoplanets. Astrophys. J. 669, 1279-1297.

Senshu, H., Kuramoto, K., Matsui, T., 2002. Thermal evolution of a growing Mars. J. Geophys. Res. 107, 5118.

Sohl, F., Spohn, T., Breuer, D., Nagel, K., 2002. Implications from Galileo observations on the interior structure and chemistry of the Galilean satellites. Icarus 157, 104-119.

Sotin, C., Grasset, O., Mocquet, A., 2007. Mass-radius curve for extrasolar Earth-like planets and ocean planets. Icarus 191, 337-351.

Stacey, F. D., 1999. Equations of state for close-packed materials at high pressures: geophysical evidence. J. Phys.: Condens. Matter 11, 575-582.

Stacey, F. D., 2000. The $K$-primed approach to high-pressure equations of state. Geophys. J. Int. 143, 621-628.

Stacey, F. D., 2001. Finite strain, thermodynamics and the Earth's core. Phys. Earth Planet. Inter. 128, 179-193.

Stacey, F. D., 2005. High pressure equations of state and planetary interiors. Rep. Prog. Phys. 68, 341-383.

Stacey, F. D., Davis, P. M., 2004. High pressure equations of state with application to lower mantle and core. Phys. Earth Planet. Inter. 142, 137184.

Stacey, F. D., Davis, P. M., 2008. Physics of the Earth. Cambridge University Press. 
Tateno, S., Hirose, K., Sata, N., Ohishi, Y., 2009. Determination of postperovskite phase transition boundary up to $4400 \mathrm{~K}$ and implications for thermal structure in D" layer. Earth Planet. Sci. Lett. 277, 130-136.

Tozer, D. C., 1972. The present thermal state of the terrestrial planets. Phys. Earth Planet. Int. 6, 182-197.

Tsuchiya, T., Tsuchiya, J., Umemoto, K., Wentzcovitch, R. M., 2004. Phase transition in $\mathrm{MgSiO}_{3}$ perovskite in the Earth's lower mantle. Earth Planet. Sci. Lett. 224, 241-248.

Turcotte, D. L., Schubert, G., 2002. Geodynamics. Cambridge University Press.

Umemoto, K., Wentzcovitch, R. M., Allen, P. B., 2006. Dissociation of $\mathrm{MgSiO}_{3}$ in the cores of gas giants and terrestrial exoplanets. Science 311, 983-986.

Valencia, D., O'Connell, R. J., Sasselov, D. D., 2006. Internal structure of massive terrestrial planets. Icarus $181,545-554$.

Valencia, D., Sasselov, D. D., O'Connell, R. J., 2007a. Detailed models of super-Earths: How well can we infer bulk properties. Astrophys. J. 665, $1413-1420$.

Valencia, D., Sasselov, D. D., O'Connell, R. J., 2007b. Radius and structure models of the first super-Earth planet. Astrophys. J. 656, 545-551.

Vinet, P., Rose, J. H., Ferrante, J., Smith, J. R., 1989. Universal features of the equation of state of solids. J. Phys.: Condens. Matter 1, 1941-1963. 


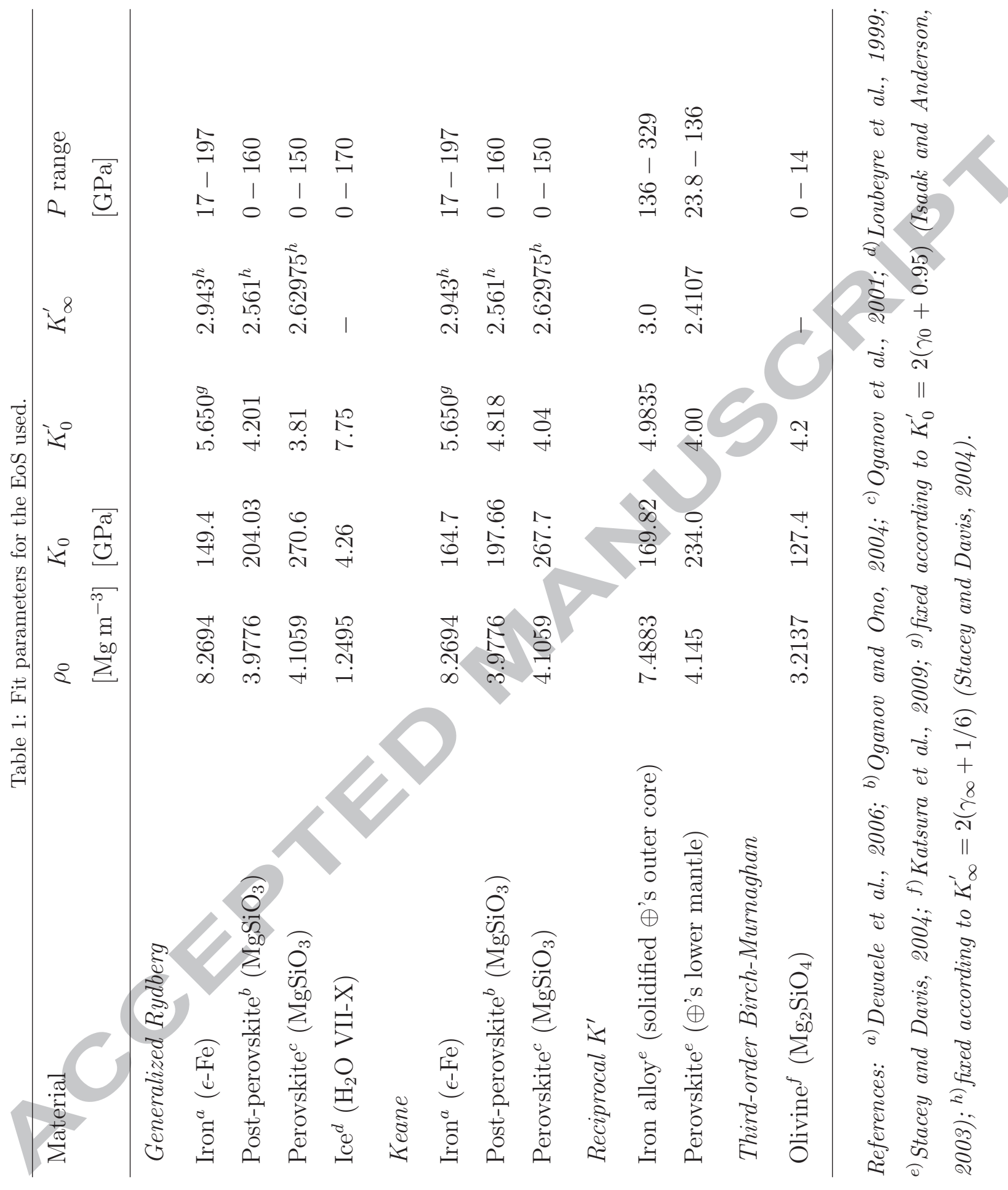


Table 2: Parameters used for the thermal correction according to Mie-Grüneisen-Debye.

\begin{tabular}{|c|c|c|c|c|}
\hline Material & $\gamma_{0}$ & $\gamma_{\infty}$ & $\beta$ & $\theta_{0}[\mathrm{~K}]$ \\
\hline \multicolumn{5}{|l|}{ Generalized Rydberg \& Keane } \\
\hline $\operatorname{Iron}^{a}(\epsilon-\mathrm{Fe})$ & 1.875 & 1.305 & $3.289^{e}$ & $430^{g}$ \\
\hline Post-perovskite $^{b}\left(\mathrm{MgSiO}_{3}\right)$ & 1.553 & 1.114 & 4.731 & $1100^{h}$ \\
\hline Perovskite $^{b}\left(\mathrm{MgSiO}_{3}\right)$ & 1.506 & 1.14821 & 7.02469 & \\
\hline \multicolumn{5}{|l|}{ Reciprocal $K^{\prime}$} \\
\hline Iron alloy ${ }^{c}$ (solidified $\oplus^{\prime}$ 's outer core $)$ & 1.8345 & 1.3333 & 3.5( & $430^{g}$ \\
\hline Perovskite $^{c}\left(\oplus^{\prime}\right.$ 's lower mantle) & 1.4545 & 1.0387 & $4.460^{f}$ & $1114^{h}$ \\
\hline \multicolumn{5}{|l|}{ Third-order Birch-Murnaghan } \\
\hline Olivine $^{d}\left(\mathrm{Mg}_{2} \mathrm{SiO}_{4}\right)$ & 1.31 & & - & 760 \\
\hline
\end{tabular}

No thermal correction has been calculated for the water-ice layer.

References: ${ }^{a)}$ Dewaele et al., 2006; ${ }^{b)}$ Ono and Oganov, 2005; ${ }^{c)}$ Stacey and Davis, 2004; ${ }^{d)}$ Katsura et al., 2009; e) assuming $\beta=\gamma_{0} /\left(\gamma_{0}-\gamma_{\infty}\right)$; ${ }^{f)}$ calculated according to $\beta=\lambda_{0} \gamma_{0} /\left(\gamma_{0}-\gamma_{\infty}\right) ;{ }^{g)}$ Dubrovinsky et al., 2000; ${ }^{h)}$ Tsuchiya et al., 2004. 
Table 3: Scaling exponent $\beta$ for low-mass exoplanets $\left(1-10 \mathrm{M}_{\oplus}\right)$.

\begin{tabular}{llll}
\hline Composition & Valencia et al. (2007a) & Sotin et al. (2007) & this study \\
\hline Earth-like & 0.262 & 0.274 & 0.267 \\
Ocean planet & 0.244 & 0.275 & 0.261 \\
Mercury-type & - & - & 0.269
\end{tabular}

Note: $R / R_{\oplus} \propto\left(M / M_{\oplus}\right)^{\beta}$. 
Figure 1: Depth-dependent interior structure of $1 \mathrm{M}_{\oplus}$ models and a reference for comparison: (a) the radial distribution of density, (b) the radial distribution of acceleration of gravity, (c) the radial distribution of hydrostatic pressure, and (d) the radial distribution of temperature. The different line styles indicate the choosen EoS, whereas the light gray dashed lines denote the reference model.

Figure 2: Depth-dependent interior structure of Earth-like exoplanets: (a) the radial distribution of density, (b) the radial distribution of acceleration of gravity, (c) the radial distribution of hydrostatic pressure, and (d) the radial distribution of temperature. From top to bottom the curves represent planets with 10 and $5 \mathrm{M}_{\oplus}$, respectively. The solid black lines correspond to the generalized Rydberg models, whereas the dashed black lines correspond to the Keane models and the solid gray lines to the reciprocal $K^{\prime}$ models.

Figure 3: (a) Mantle viscosity corresponding to the models using the generalized Rydberg EoS. (b) Creep activation volume $V^{*}$ as a function of pressure. Note that $V^{*}$ scales nonlinearly with pressure $P$ that is given in units of $\mathrm{Pa}$ in the inset scaling law. 
Figure 4: Mass-radius relationships of low-mass exoplanets ranging from 1 to $15 \mathrm{M}_{\oplus}$. The solid curves are homogeneous, self-compressible spheres of the following materials: waterice (blue line), Mg-perovskite (red line), and $\epsilon$-Fe (black line). The dashed curves denote differentiated planets of various bulk composition. The red dashed curve is for Earth-like planets with an iron core of $32.5 \mathrm{wt} .-\%$ and a $67.5 \mathrm{wt} .-\%$ silicate mantle. The blue curve is for ocean planets using the Jovian moon Ganymede as a type-example, resulting in a 45 wt.- $\%$ water-ice shell surrounding a 48.5 wt. $\%$ silicate mantle, and a 6.5 wt.- $\%$ iron core. The black dashed curve is for iron-rich planets like Mercury composed of a 70 wt.- $\%$ iron core overlain by a $30 \mathrm{wt} .-\%$ silicate mantle. The triangle indicates the relative position of the Earth. The solid ellipses represent the first low-mass exoplanets with a measured planetary radius and mass according to their observational uncertainties. 

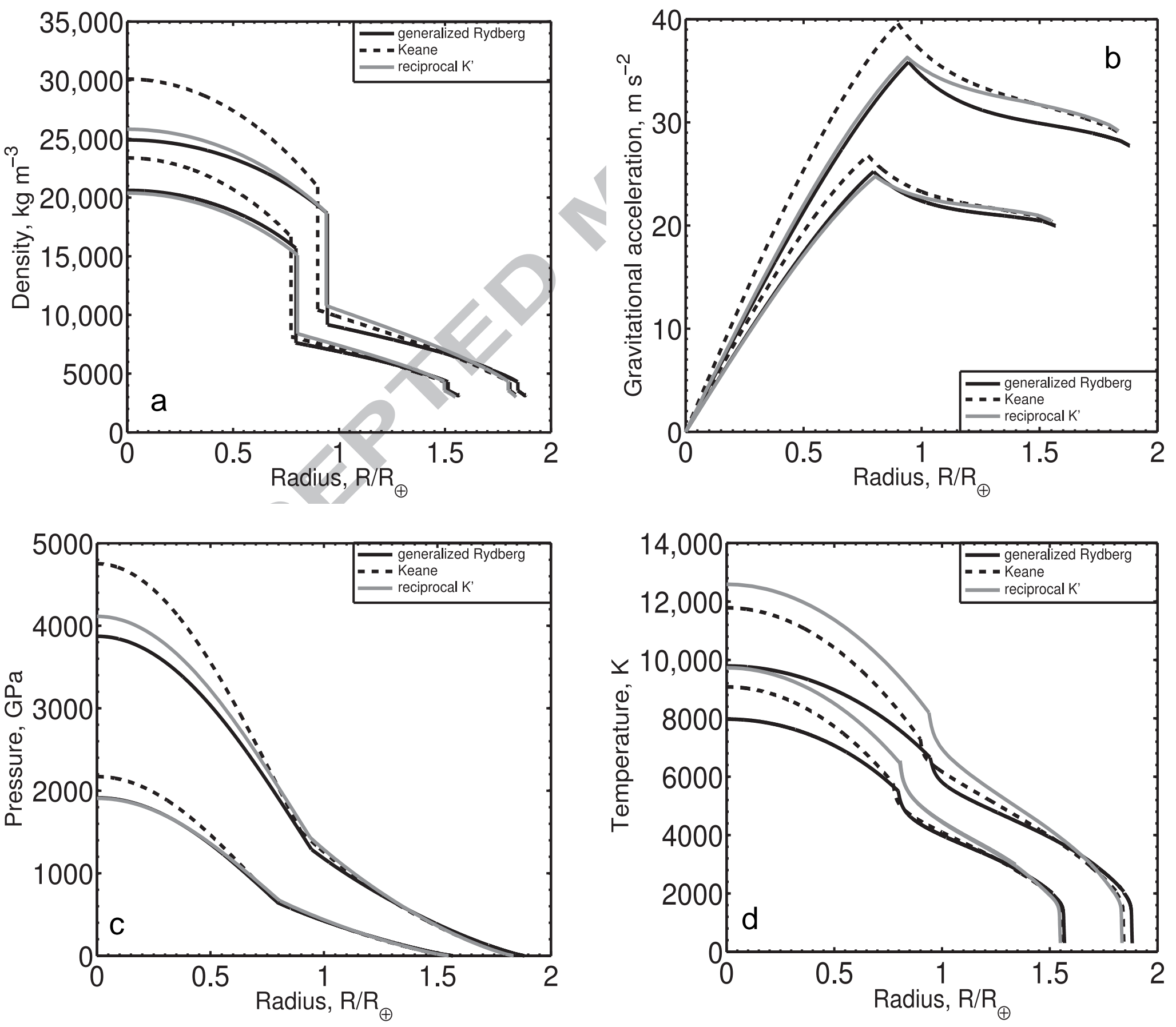


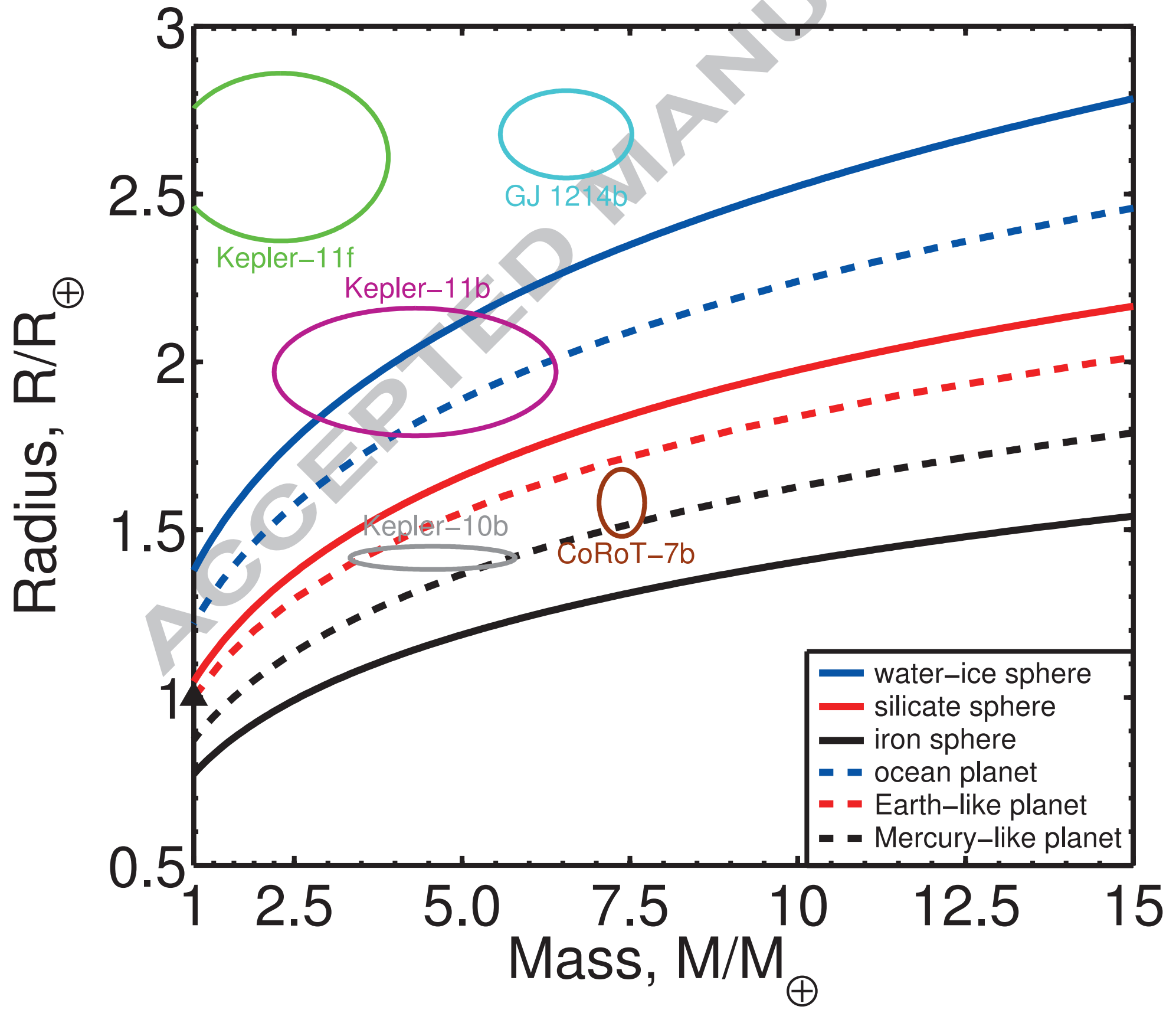




\section{Research Highlights}

Structural models of solid exoplanet interiors are constructed using equations of state for the radial density distribution, which are compliant with the thermodynamics of the high-pressure limit. Trade-offs in predicted radii of terrestrial-type exoplanets of up to ten Earth masses fall well within current observational limits. Deep exoplanet interiors are likely hotter than previously thought because of the pressure-induced, less vigorous convective heat transfer at depth. 